\title{
A Single Atrial Extrastimulus Can Distinguish Sinus Tachycardia from 1:1 Paroxysmal Tachycardia
}

\author{
Tachycardia Sinusale et Tachycardie Paroxystique: Distinction \\ par un Seul Extrastimulus Auriculaire
}

\author{
JANICE JENKINS, * KI HONG NOH,* THOMAS BUMP, \\ FRANCES MUNKENBECK, JEFFREY BROWN, and ROBERT ARZBAECHER
}

From the Illinois Institute of Technology, and University of Chicago, Chicago, Illinois, and

*The University of Michigan, Ann Arbor, Michigan

\begin{abstract}
JENKINS, J., ET AL.: A single atrial extrastimulus can distinguish sinus tachycardia from 1:1 paroxysmal tachycardia. We have developed a tachycardia detection scheme for use in an antitachycardia pacemaker in which the use of a properly timed atrial extrastimulus provides a means of discriminating sinus tachycardia from pace-terminable 1:1 tachycardias. An atrial extrastimulus is delivered in late diastole (80 ms premature), and the ventricular response is monitored. In sinus tachycardia, the ventricular response is expected to appear early as well, but in pace-terminable tachycardias, such as AV reentrant and ventricular with VA conduction, the ventricular rhythm will be unperturbed. Testing of the algorithm was performed in 34 patients. In 29 patients, atrial extrastimuli were delivered during sinus tachycardia, and in 22 patients during various types of 1:1 paroxysmal tachycardia. In one patient the procedure was completely automated, i.e., delivery of the atrial extrastimuli and diagnosis were microcomputer controlled. In 28/29 cases, the delivery of an atrial extrastimulus 80 to $120 \mathrm{~ms}$ early during sinus tachycardia elicited a ventricular response at least $28 \mathrm{~ms}$ early. In 22/22 patients with 1:1 paroxysmal tachycardia, atrial extrastimuli 80 to $120 \mathrm{~ms}$ early failed to produce a significant change in ventricular cycle length. This technique appears to be promising for prevention of inadvertent pacing of sinus tachycardia in an antitachycardia pacemaker. (PACE, Vol. 9, November-December, Part II, 1986)
\end{abstract}

antitachycardia devices, atrial extrastimulus, detection algorithms

\section{Introduction}

Electrical management of supraventricular tachycardia is becoming an increasingly important therapeutic tool, and the use of implantable devices employing microprocessor technology has served to advance this therapy. The reliable dis-

This work was supported in part by a grant from the National Institutes of Health, HL-35554, and a grant from the National Science Foundation, ECS-8351215.

Address for reprints: Janice M. Jenkins, Ph.D., Department of Electrical Engineering and Computer Science, The University of Michigan, Ann Arbor, MI 49109. tinction of sinus tachycardia from clinical tachycardias remains a major limitation of these devices. $^{1,2}$ The use of suddenness of onset for recognition of paroxysmal tachycardia has been proposed $^{3,4}$ and implemented with some success. ${ }^{5}$ Yet many paroxysmal tachycardias are initiated hesitantly by isolated premature beats and reach a stabilized rate only after seven or more beats. ${ }^{6}$ By contrast, some sinus tachycardias mimic paroxysmal tachycardias in their abrupt initiation. ${ }^{2,7}$ In this paper, we describe a technique in which the pacemaker employs a provocative test for the distinction of sinus from clinical tachycardia. The device (after recognition of a sustained high rate in both chambers reflecting a 1:1 atrioventricular 
relationship) automatically delivers a precisely timed atrial extrastimulus in late diastole and uses the ventricular response as a discriminant function. Our hypothesis is that the ventricular response in sinus tachycardia should differ from that in most forms of 1:1 paroxysmal tachycardia.

\section{Background}

We have previously described an algorithm ${ }^{3,4}$ that monitors atrial and ventricular intracardiac electrodes and produces a diagnosis of supraventricular tachycardia with block, atrial flutter, or atrial fibrillation by recognizing a high rate with a preponderance of atrial signals over ventricular. In a similar manner, sequences with more ventricular than atrial deflections are easily categorized as ventricular tachycardia or fibrillation, depending on rate. In the case where there are an equivalent number of atrial and ventricular signals, we have previously employed an onset criterion that attempted to delineate sinus tachycardia from pace-terminable tachycardia on the basis of suddenness of initiation. This method, while superior to rate alone, lacks specificity in the case of rapid acceleration due to fright, stress, or excitement. ${ }^{7}$ In our present algorithm, we have introduced an atrial extrastimulus technique to provide distinction of sinus versus clinical tachycardia. The test is based on the hypothesis that a slightly early stimulation of the atria in sinus tachycardia would result in a concomitant early response in the ventricles, but the same early atrial capture would be blocked in the AV node in the case of the AV nodal tachycardia or ventricular tachycardia with VA conduction. Thus, the underlying ventricular rhythm in the latter tachycardias would be unperturbed, and a ready distinction would be available. The technique requires that the tachycardia under study have a regularity of cycle length in both chambers; otherwise, the interpretation of the response could be ambiguous. Initial studies in the electrophysiology laboratory using manual delivery of the extrastimulus and visual measurements of ventricular response have been previously published. ${ }^{7}$ We have completely automated the recognition scheme and the extrastimulus test for further testing and validation as a closed-loop control system.

\section{Methods}

Software has been implemented on an IBM PC/AT with a Lab Master** analog-to-digital (A/ D) and digital-to-analog (D/A) subsystem. Hardware triggers derived from the atrial and ventricular signals provide real time interrupts with a 4 $\mathrm{ms}$ resolution. Programming was done in C language and $8088 / 8086$ assembly language. A flow chart depicting the computer algorithm is seen in Fig. 1. The atrial extrastimulus procedure is employed only in the case of 1:1 tachycardia and is intended to recognize and exclude sinus tachycardia from attempts at termination by pacing.

Thirty-four patients undergoing electrophysiologic study at the University of Chicago were entered into the study after giving informed consent. During diagnostic electrophysiologic procedures in which quadripolar or hexapolar catheter electrodes were introduced into the right atrium, the His bundle, and right ventricular apex, standard pacing protocols (incremental atrial pacing, atrial extrastimulus, and incremental ventricular pacing) were performed via a programmable stimulator (Bloom Associates†). Surface leads I, II, III, V1, and intracardiac electrograms were recorded on an oscilloscope and paper recorder (Electronicsfor-Medicineł VR16).

In 22 cases, the patient's clinical tachycardia was induced by programmed stimulation. In 29 patients, sinus tachycardia was pharmacologically induced by the administration of atropine and/or isoproterenol. Upon confirmation of the regularity of the tachycardia in both chambers, the effect of extrastimulation of the high right atrium was evaluated. A single atrial extrastimulus (S2) was coupled to the ninth beat (A1) of the tachycardia starting with an extrastimulus delivered $80 \mathrm{~ms}$ prior to the expected atrial beat. The coupling interval was then decremented by $10 \mathrm{~ms}$ until atrial refractoriness was reached.

In one patient, the tachycardia detection scheme and invocation of the extrastimulus procedure was completely controlled by the com-

\footnotetext{
** Scientific Solutions, Inc., Solon, OH, USA.

$†$ Bloom Associates, Narbeth, PA, USA.

‡ Electronics-for-Medicine, Pleasantville, NY, USA.
} 


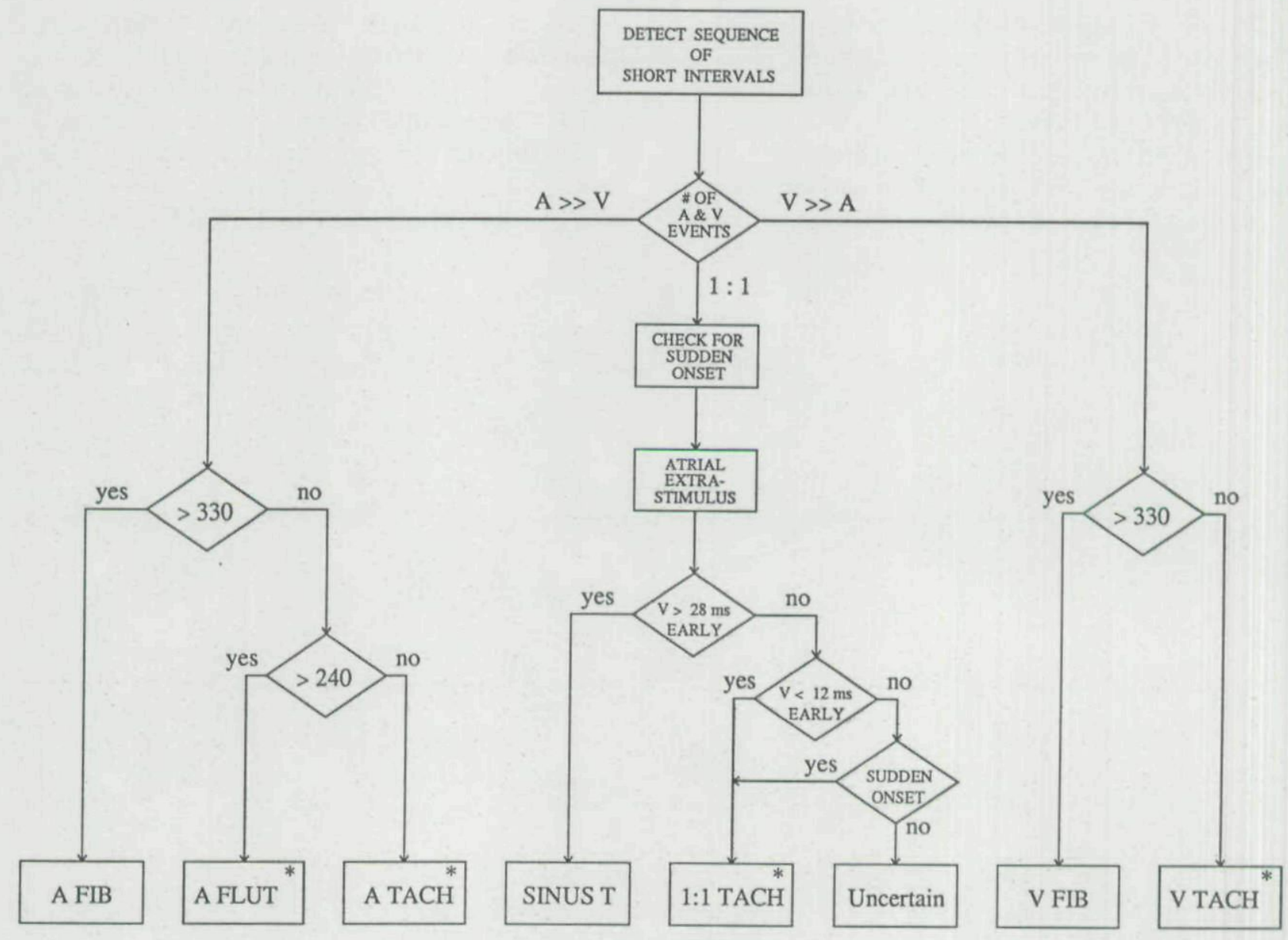

A FIB $=$ atrial fibrillation, A FLUT $=$ atrial flutter, $\mathrm{A}$ TACH $=$ atrial tachycardia, SINUS $\mathrm{T}=$ sinus tachycardia, 1:1 $\mathrm{TACH}=1: 1$ paroxysmal tachycardia, $\mathrm{V}$ FIB $=$ ventricular fibrillation, $\mathrm{V}$ TACH $=$ ventricular tachycardia.

Figure 1. Flow chart of the tachycardia detection scheme and atrial extrastimulus test. To avoid inadvertent pacing during sinus tachycardia, a provocative test is invoked upon recognition of a 1:1 tachycardia. The premature ventricular response to an early (late diastole) atrial extrastimulus provides discrimination of sinus tachycardia from 1:1 clinical tachycardias. Arrhythmias marked with asterisks are considered pace-terminable.

puter. The subject, a 14-year-old female presenting with AV nodal tachycardia, was paced using programmed stimulation until the clinical tachycardia was induced. Upon exceeding a rate of 120 bpm for eight consecutive beats, the tachycardia was computer detected, and a diagnosis of 1:1 tachycardia was reported. Atrial extrastimulation was performed according to the protocol described above for five separate trials in which coupling intervals ranged from 80 to $120 \mathrm{~ms}$ early. The ven- tricular response was monitored by computer and the associated VV interval compared with the immediately preceding VV interval in the tachycardia. If the ventricular response was found to be $>28$ ms early, a diagnosis of sinus tachycardia would be reported. If the response was $<12 \mathrm{~ms}$ early the diagnosis would be paroxysmal tachycardia suitable for pace-termination. If the ventricular response fell between 12 and $28 \mathrm{~ms}$, the result is considered indeterminate, and the earlier 
onset information is invoked for a decision rule. Thus the onset criterion is employed only as a back-up measure should the atrial extrastimulus test result in uncertainty.

In all extrastimulus trials during AV nodal tachycardia, the ventricular response fell below 12 ms early. Figure 2 shows an atrial extrastimulus delivered $120 \mathrm{~ms}$ early and a subsequent ventricular beat which occurs within $4 \mathrm{~ms}$ of the previous VV interval. The computer diagnosis of paroxysmal 1:1 tachycardia is given.

Following conversion of the clinical tachycardia, the patient was studied during sinus tachycardia induced by atropine. The same pro-

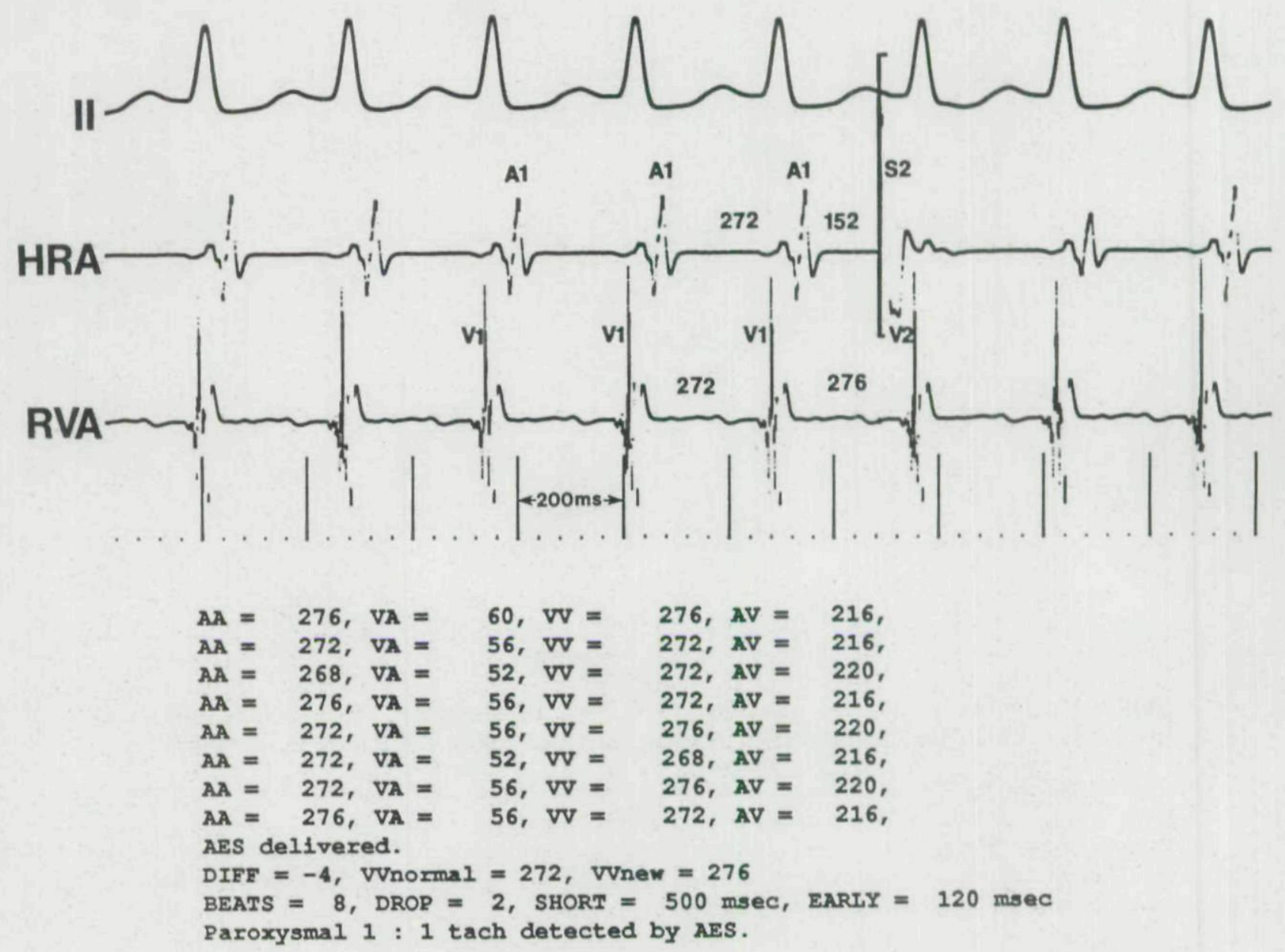

Figure 2. Atrial extrastimulus delivered during AV nodal reentrant tachycardia. The top channel is lead II, the middle is high right atrial (HRA), and the bottom, right ventricular apex (RVA). Time lines are generated at $200 \mathrm{~ms}$. During the tachycardia, which has a predominant cycle length of $272 \mathrm{~ms}$, an atrial extrastimulus (52) is delivered $120 \mathrm{~ms}$ premature but causes no effective change in ventricular rhythm. The computer listing is shown beneath the tracing. Reading from left to right, upon recognition of an atrial signal, the corresponding AA interval and related VA interval are reported. In a similar manner, the ventricular signals are detected and VV and AV intervals reported. When eight short intervals $(>120 \mathrm{bpm})$ in either chamber are seen, a computer diagnosis is reported. In the case of 1:1 tachycardias, an atrial extrastimulus (AES) is delivered and ventricular response (VV new) compared to the ventricular rate during the tachycardia (VV normal). If no significant change occurs in the ventricular rhythm, a diagnosis of PAROXYSMAL 1:1 TACHYCARDIA is reported. (See flow chart in Fig. 1). 
tocol was performed during sinus tachycardia in which regularity was confirmed and atrial extrastimulation was introduced at intervals 80 to 120 ms early. The ventricular response was monitored by computer and a computer diagnosis delivered. In all five extrastimulus attempts, the ventricular response was greater than $28 \mathrm{~ms}$ early. Figure 3 shows a tracing of an atrial extrastimulus delivered $120 \mathrm{~ms}$ early during sinus tachycardia. The coupling interval is $236 \mathrm{~ms}$ and the new VV interval is determined by computer to be $288 \mathrm{~ms}$ or $68 \mathrm{~ms}$ early.

\section{Results}

Twenty-two patients were studied using the atrial extrastimulus procedure during various types of 1:1 clinical tachycardias. In 29 patients, sinus tachycardia was induced as described. In one patient, the procedure was completely automated, i.e., arrhythmia detection, delivery of atrial extrastimuli, and diagnosis were microprocessor controlled. In 28/29 cases, the delivery of an atrial extrastimulus 80 to $120 \mathrm{~ms}$ early in sinus tachycardia elicited a ventricular response at least 28

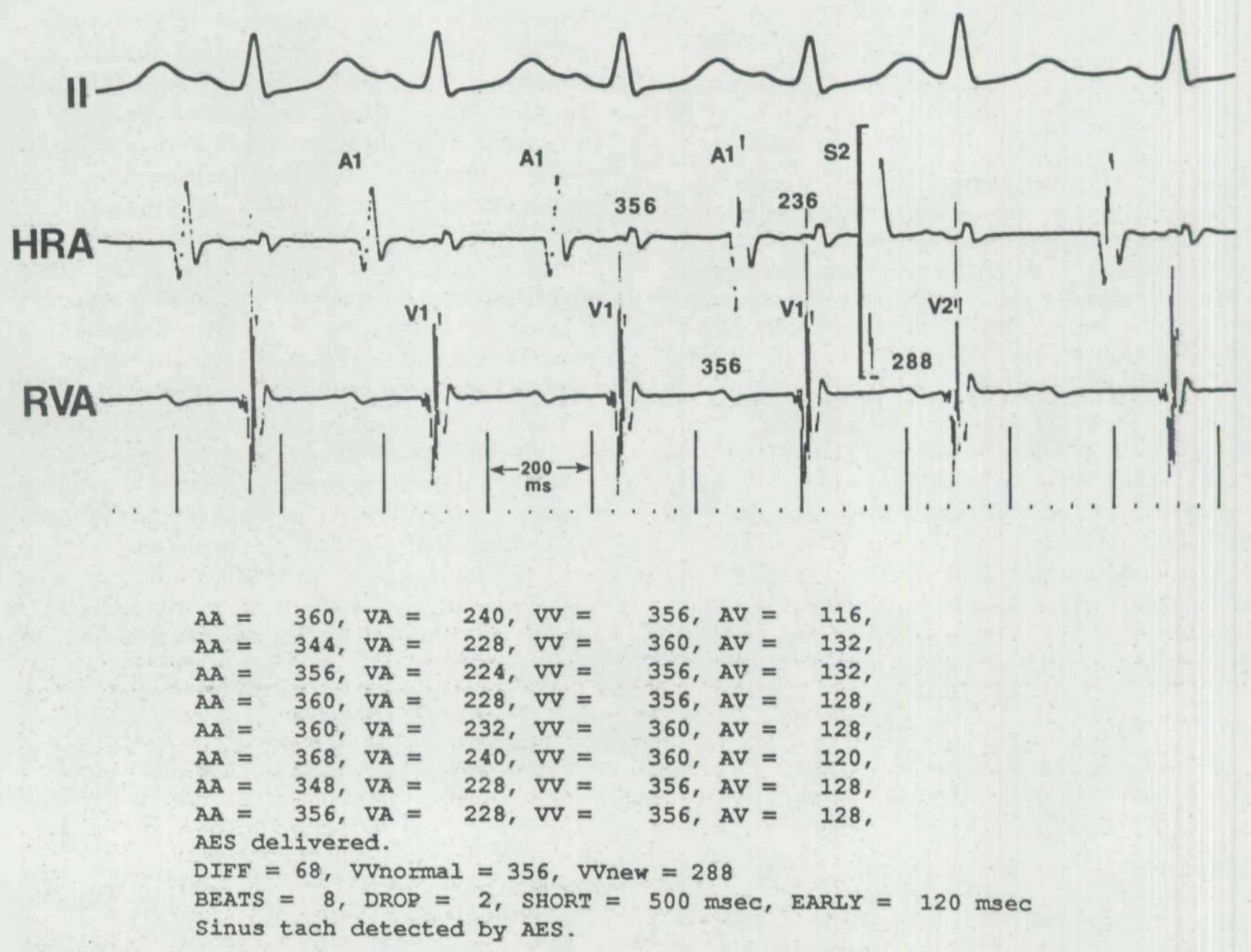

Figure 3. Atrial extrastimulus delivered during sinus tachycardia. The figure is ordered as in Fig. 2 and depicts the same patient. During the tachycardia, which has a predominant cycle length of $356 \mathrm{~ms}$, an atrial extrastimulus is delivered $120 \mathrm{~ms}$ premature resulting in the subsequent ventricular beat being $68 \mathrm{~ms}$ premature. The computer diagnosis of SINUS TACHYCARDIA is given. 


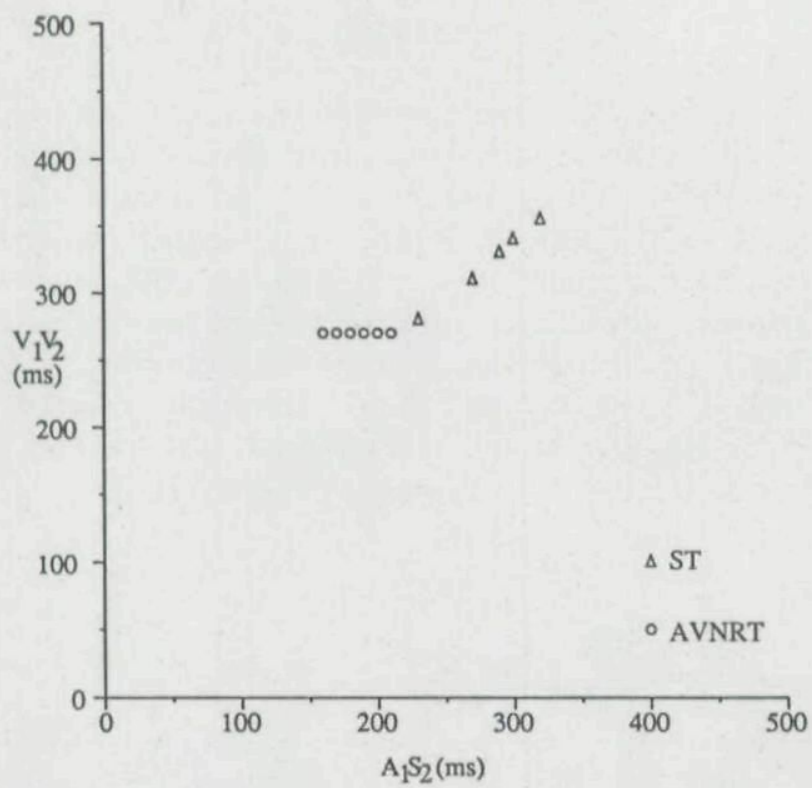

Figure 4. A1S2, V1V2 curve representing single atrial extrastimulation during the patient's sinus tachycardia (ST) and AV nodal reentrant tachycardia (AVNRT). Results obtained during sinus tachycardia are indicated by the open triangles, and the results during the paroxysmal tachycardia are indicated by the open circles. AVNRT cycle length $=270 \mathrm{~ms}$; ST cycle length $=360 \mathrm{~ms}$. See text for further discussion.

ms early. In 22/22 patients with 1:1 paroxysmal tachycardia, atrial extrastimuli 80 to $120 \mathrm{~ms}$ early failed to produce a ventricular response more than $12 \mathrm{~ms}$ premature.

A conduction curve obtained during both sinus and paroxysmal tachycardia from the patient who was subject to computer monitoring and control of the extrastimulus test is shown in Fig. 4. In

\section{References}

1. Fisher, J.D., Kim, S.G., Furman, S., Matos, J.A.: Role of implantable pacemakers in control of recurrent ventricular tachycardia. Am. J. Cardiol., 49:194, 1982.

2. Fisher, J.D., Goldtein, M., Ostrow, E., Mator, J.A., Kim, S.G.: Maximal rate of tachycardia development: Sinus tachycardia with sudden exercise vs. spontaneous ventricular tachycardia. PACE, 6:221, 1983.

3. Arzbaecher, R., Bump, T., Jenkins, J., Glick, K., Munkenbeck, F., Brown, J., Nandhakumar, N.: Automatic tachycardia recognition. PACE, 7:541, 1984.

4. Jenkins, J., Bump, T., Munkenbeck, F., Brown, J., Arzbaecher, R.: Tachycardia detection in implantable antitachycardia devices. PACE, 7:1273, 1984. this case, the patient had an AV nodal reentrant tachycardia with cycle length 272 . The atropine induced sinus tachycardia had cycle length 356. Results show that during sinus tachycardia, as the atrial extrastimulus was delivered at decreasing cycle lengths, the ventricular response was correspondingly foreshortened. By contrast, during the 1:1 AV nodal tachycardia, the introduction of increasingly early atrial extrastimuli produced no significant change in ventricular cycle length.

\section{Discussion}

The provocative use of a properly timed atrial extrastimulus by an antitachycardia pacemaker provides a means of discrimination of difficult 1: 1 tachycardias. Specific identification of sinus tachycardia is critical if inadvertent pacing is to be prevented. While onset criteria may be accurate in diagnosing true paroxysmal tachycardias, it has been found to misclassify many sinus tachycardias as paroxysmal, thus subjecting patients to unwarranted pacing. The addition of the atrial extrastimulus test to the diagnostic computer logic provides improved specificity in the recognition of sinus tachycardia. The computer algorithm for tachycardia recognition and automated delivery of atrial extrastimuli to distinguish 1:1 tachycardias has been successfully demonstrated in a patient during electrophysiology study in a catheterization laboratory. It should be pointed out that the tachycardia under study must possess regularity if the interpretation of the response is to be unambiguous. Given this caveat, the algorithm appears to be promising for use in an antitachycardia device.

5. Pless, B.D., Sweeney, M.B.: Discrimination of supraventricular tachycardia from sinus tachycardia of overlapping cycle length. PACE, 7:1318, 1984.

6. Olson, W.H., Bardy, G.H.: Cycle length and morphology pattern at the onset of spontaneous ventricular tachycardia and fibrillation. 7th Annual Scientific Session NASPE, 1986, p. 284.

7. Brown, J.P., Gillette, P.C., Goh, T.H., Arzbaecher, R.: Discrimination of tachycardia by rate of onset. Engineering Foundation Computer Interpretation of the ECG XI, 1986.

8. Munkenbeck, F., Bump, T., Arzbaecher, R.: Differentiation of sinus tachycardia from paroxysmal 1:1 tachycardia using single late diastolic atrial extrastimuli. PACE, 9:53, 1986. 
This document is a scanned copy of a printed document. No warranty is given about the accuracy of the copy. Users should refer to the original published version of the material. 